\title{
Gedanken zu einem Präventivgesetz 1
}

\author{
Martha Ribi ${ }^{2}$
}

Gruppe: Gesetzgebung. Gruppenleitung: Nationalrätin Martha Ribi, Zürich. Referenten: Dr. med. U. Frey, Bern; Dr. med. J. Martin, Lausanne.

Die Gruppe «Gesetzgebung» stellte sich nach einleitenden Referaten über die drei Bereiche der primären, sekundären und tertiären Prävention (Dr. med. $J$. Martin) und über ein in Vorbereitung befindliches eidgenössisches Präventivgesetz (Dr. med. U. Frey) die grundsätzliche Frage, ob eine gesetzliche Regelung überhaupt notwendig sei und welche Voraussetzungen sie allenfalls zu erfüllen hätte. Folgendes wurde dabei festgehalten:

1. Es soll ein Rahmengesetz sein, das die Kompetenz der für das Gesundheitswesen zuständigen Kantone nicht einschränkt.

2. Es soll weder ein Subventionierungs- noch ein Polizeigesetz sein.

3. Man verspricht sich von einem solchen Gesetz eine Symbolwirkung.

4. Das Gesetz muss sich auf Realisierbares in der Prävention beschränken, sonst ist es politisch nicht durchzubringen.

Gestützt auf diese Gedanken und auf eine eingehende Aussprache zwischen den Teilnehmern der Gruppe wurden die folgenden Gedanken zu einem Präventivgesetz formuliert:

\section{Zweck}

Schutz und Förderung der Gesundheit durch erzieherische und vorbeugende Massnahmen. Vorbehalten bleibt die Koordination mit den bereits bestehenden Bundesgesetzen im relevanten Bereich.

\section{Geltungsbereich}

Gesundheitliche Risiken, die Krankheiten, Gebrechen und Unfälle verursachen können, die wegen ihrer Schwere, Häufigkeit, Invalidisierungsfolge und finanziellen Folgen für den einzelnen, seine Familie und/ oder die Gesellschaft besonders schwerwiegend sind und durch präventive Massnahmen verhütet oder günstig beeinflusst werden können.

Insbesondere erstreckt sich der Geltungsbereich auf

- Missbrauch von Alkohol, Tabak, Medikamenten und Betäubungsmitteln

- andere Gesundheitsrisiken, wie Fehlernährung, Bewegungsmangel

- Zahnhygiene

- Psychohygiene

1 Bericht der Arbeitsgruppe “Gesetzgebung», Forum Davos 78: Grenzen der Medizin III: Prävention und ihre Möglichkeiten.

2 Lic. oec. publ. Nationalrätin, Kilchbergstrasse 45, 8038 Zürich.
3. Aufgaben der Kantone

- Förderung der Gesundheitserziehung

Aufklärung besonders gefährdeter Bevölkerungsgruppen

Verbesserung der humanökologischen Umweltbedingungen

- Förderung der Aus- und Weiterbildung von Fachpersonal.

Die interkantonale Zusammenarbeit ist anzustreben

\section{Aufgaben des Bundes}

- Der Bund unterstützt Kantone und private Organisationen durch Dienstleistungen (z. B. Statistiken, Dokumentation, Information).

- Er fördert und koordiniert die Tätigkeit der Kantone und der privaten Organisationen auf nationaler Ebene.

- Er kann ein konsultatives Koordinationsorgan einsetzen, wobei bestehende Kommissionen zu berücksichtigen sind.

- Der Bund kann gesamtschweizerische Vorbeugungsmassnahmen in Zusammenarbeit mit den Kantonen und privaten Organisationen durchführen.

- Der Bund fördert die wissenschaftliche Forschung über gesundheitserzieherische und andere präventive Massnahmen.

\section{Private Organisationen}

Bund und Kantone können in gegenseitigem Einvernehmen bestimmte Aufgaben und Befugnisse privaten Organisationen übertragen.

\section{Finanzen}

Für die dem Bund zu übertragenden Aufgaben sind angemessene Mittel im Budget einzustellen. Es soll untersucht werden, wie gross der Aufwand sein wird und ob allenfalls neue Finanzierungsquellen erschlossen werden müssen.

Das Verursacherprinzip soll angemessen berücksichtigt werden.

\section{Summary}

Propositions for a "law for prevention"

The proposed law should concentrate on prophylaxis and educational measures aimed at diseases of public health importance. It should stimulate action on the federal, cantonal and private level and the cooperation of all parties concerned. Where feasible and adequate, finances should be raised from those making preventive and curative actions necessary.

\section{Teilnehmerliste der Arbeitsgruppe "Gesetzgebung"}

P. Calpini, Sion; U. Frey (Experte), Bern; A. Gautier, Genf; Marianne Gerber, Bern; J. Gross, Weinfelden; P. Guetg, Zürich; U. Krieger, Basel; P. Manz, Liestal; J. Martin (Experte), Lausanne; Martha Ribi (Vorsitz), Zürich; Chr. Richner, Winterthur; H. Rittmeyer, Zürich; Chr. E. Schultz, Basel; W. P. von Wartburg, Basel. 